\title{
Projecting Biochemistry Over Long Distances
}

\author{
M. Reed ${ }^{1}$, H. F. Nijhout ${ }^{2}$, J. Best ${ }^{3}$ \\ ${ }^{1}$ Department of Mathematics, Duke University, Durham, NC 27705, USA \\ 2 Department of Biology, Duke University, Durham, NC 27705, USA \\ ${ }^{3}$ Department of Mathematics, The Ohio State University, Columbus, OH 43210, USA
}

\begin{abstract}
Mathematical and computational neuroscience have contributed to the brain sciences by the study of the dynamics of individual neurons and more recently the study of the dynamics of electrophysiological networks. Often these studies treat individual neurons as points or the nodes in networks and the biochemistry of the brain appears, if at all, as some intermediate variables by which the neurons communicate with each other. In fact, many neurons change brain function not by communicating in one-to-one fashion with other neurons, but instead by projecting changes in biochemistry over long distances. This biochemical network is of crucial importance for brain function and it influences and is influenced by the more traditional electrophysiological networks. Understanding how biochemical networks interact with electrophysiological networks to produce brain function both in health and disease poses new challenges for mathematical neuroscience.
\end{abstract}

Keywords and phrases: networks, electrophysiology, biochemistry, brain

Mathematics Subject Classification: 92

\section{A tribute to Mike Mackey}

No one has had more influence on the development of mathematical biology over the past 40 years than Michael Mackey. No one. There are three reasons. First, his own research program is very broad, extending from clinical applications [16] to theoretical mathematics [14][19]. The focus on medicine provided an important example to mathematical biologists who see biology only as a source of new mathematical problems. His theoretical work showed the pure mathematics community how much new mathematics would come from biology. Secondly, Mike saw very early that a new kind of interdisciplinary education was necessary for undergraduates. He was instrumental in establishing in 1974 a program in "Physiology and Physics" and in 1991 a program in "Physiology and Mathematics" at McGill. Thirdly, because he studied real physiological systems, he and Leon Glass understood long before the rest of us that delay differential equations [17][18][15] and stochastic analysis [14] would be fundamental for understanding the behavior of biological system at all levels.

*Corresponding author. E-mail: reed@math.duke.edu 


\section{The electrophysiological view of the brain.}

It is natural for us to think of the brain as a large computational device that processes information analogously to a computer. In this view, which we like to call the electrophysiological point of view, the basic elements are the neurons that receive inputs from other neurons and, via action potentials, send information to other neurons. There are then two fundamental classes of biological (and mathematical) questions. How do individual neurons receive and process their inputs and decide when to fire? How do connected sets of neurons perform information processing functions that individual neurons cannot do? The electrophysiological point of view is natural for two reasons. First, we have had great success in building computational machines and we understand completely how they work. If brains are like our computational devices then we can use computational algorithms as metaphors and examples of what must be going on in the brain. Secondly, the electrophysiological point of view fits well with our modern scientific method of trying to understand complex behavior in the large as merely the interaction of many fundamental parts (the neurons) whose behavior we understand very well.

The electrophysiological point of view is perfect for mathematical analysis and computation. One need not deal with the messy details of cell biology, the existence of more than 50 identified neurotransmitters, or the fact that neurons come in a bewildering variety of different morphological and physiological types. All of these things appear, if they appear at all, as parameters in models of neurons, or as parameters in local or global network simulations. In particular, the chemistry of neurotransmitters themselves is not very important, since their only role is to help the electrophysiological brain transmit information from one neuron to the next.

\section{Volume transmission.}

It has been known for a long time [9] that not all neurons are engaged in the one-to-one transfer of information to other neurons. Instead, groups of neurons that have the same neurotransmitter can project densely to a distant volume (a nucleus or part of a nucleus) in the brain and when they fire they increase the concentration of the neurotransmitter in the extracellular space in the distant volume. This increased concentration modulates the electrophysiological neural transmission in the distant region by binding to receptors on the cells in the target region. This kind of neural activity is called volume transmission. It is also called neuromodulation because the effect of the neurotransmitter is not one-toone neural transmission but instead the modulation of other transmitters that are involved in one-to-one transmission.

Some examples of volume transmission are the dopaminergic projection to the striatum in the basal ganglia from the cells of the substantial nigra pars compacta ( $\mathrm{SNc}$ ) and the serotonergic projection to the striatum from the dorsal raphe nucleus (DRN). These examples are discussed further below. Projections of norepinepherine (NE) neurons from the locus coeruleus to the cortex play an important role in initiating and maintaining wakefulness. This cell group globally innervates large parts of the brain and the spinal cord and gives rise to fine varicose NE terminal networks of low to moderate densities present all over the cerebral and cerebellar cortices [10].

\section{The pharmacological view of the brain.}

There are many pieces of evidence that suggest that volume transmission plays a fundamental role in the functioning of the brain. Dopamine (DA) has been linked to fundamental brain functions such as motivation, pleasure, cognition, memory, learning, and fine motor control, as well as social phobia, Tourette's syndrome, Parkinson's disease, schizophrenia, and attention deficit hyperactivity disorder [9]. In most experiments it is the concentration of dopamine in a particular nucleus that is important. Similarly, serotonin (5HT) has been linked to feeding and body weight regulation, aggression and suicidality, social hierarchies, obsessive compulsive disorder, alcoholism, anxiety disorders, and affective disorders such as depression. Many pharmaceutical drugs and recreational drugs have been shown to act by binding to 
certain receptors and thus changing the the local concentrations of various neurotransmitters in local regions of the brain. For example, the immediate effect of selective serotonin reuptake inhibitors (SSRIs) is to inhibit the reuptake of $5 \mathrm{HT}$ after it has been released thus increasing its concentration in the extracellular space in certain brain regions. Adenosine is an important neuromodulator [6]. Caffeine binds to adenosine receptors and cocaine blocks the reuptake of DA, 5HT, and norepinepherine [9].

Furthermore, various morphological and physiological features of the brain are consistent within the idea that the purpose of some neurons is to change the local biochemistry at distant regions of the brain. Often the projections are dense in the target volume suggesting that the idea is to change the local concentration at all parts of the target region simultaneously by the same amount. There are 50 or more types of receptors for $5 \mathrm{HT}$ in the brain [1], suggesting that this great variety allows the concentration of $5 \mathrm{HT}$ to modulate neurons in different ways depending on what receptors they express. The $5 \mathrm{HT}$ neurons in the dorsal raphe nucleus (DRN) have very thin unmyelinated axons and release $5 \mathrm{HT}$ from many small varicosities [13] rather than synapses, suggesting that their purpose is not one-to-one neural transmission. 5HT neurons in different parts of the DRN project to many different brain regions that frequently project back [20], suggesting that the DRN is differentially changing the local biochemistry in many distinct regions. There is a long list of different DA receptors. For example, in the striatum, the DA projections from the SNc inhibit medium spiny neurons in the indirect pathway by binding to D2 receptors while the same neurons excite medium spiny neurons in the direct pathway by binding to D1 receptors.

Notice that what is important in volume transmission is that local groups of neurons project to distant nuclei and change the local biochemistry there. That is, they project changes in biochemistry over long distances. Of course they do this by firing action potentials. But the action potentials do not carry information in the usual sense; their only purpose is to allow the neurons to project biochemistry over long distances. This is the pharmacological view of the brain. For an excellent recent review with a historical perspective and many examples, see [10].

\section{Dopamine and serotonin in the striatum.}

To illustrate both the electrophysiological view and the pharmacological view, and how they interact, we will describe a circuit that we have have recently studied [21]. Figure 1 shows schematically two circuits, the direct and indirect pathways from the cortex to the striatum and then to the thalamus. Some intermediate steps have been left out. The direct pathway excites the thalamus and the indirect pathway inhibits the thalamus. Dopaminergic neurons in the SNc project to the striatum and release DA that excites neurons in the direct pathway and inhibits neurons in the indirect pathway (indicated by the plus and minus signs in Figure 1).

In Parkinson's disease, cells in the SNc die, so less DA is released in the striatum; the result is that the direct pathway is less excited and the indirect pathway is less inhibited, so the thalamus receives more inhibition and less excitation. In the standard theory of Parkinson's disease, this imbalance between the direct and indirect pathways is thought to result in bradykinesia and rigidity [22]. The projection from the SNc to the striatum is very dense and the evidence is strong that it is the DA concentration in the extracellular space that is important for keeping the balance between the direct and indirect pathways, not one-to-one neural transmission. In particular, DA agonists given to Parkinson's patients are somewhat successful in restoring function [4]. Thus, DA, projected from the SNc, is acting as a neuromodulator of the direct and indirect pathways.

However, the situation is even more interesting. The dorsal raphe nucleus (DRN) contains 5HT neurons that also make a dense projection to the striatum. When these neurons fire they release $5 \mathrm{HT}$ into the extracellular space in the striatum. The higher the concentration of $5 \mathrm{HT}$ in the striatum, the more DA is released from the DA neurons projecting from the SNc per action potential [2][3][7]. Thus, DA is acting as a neuromodulator in the striatum and the release of DA itself is being modulated by another neuromodulator, $5 \mathrm{HT}$, that is being projected from the DRN. Only one input to the DRN is pictured in 


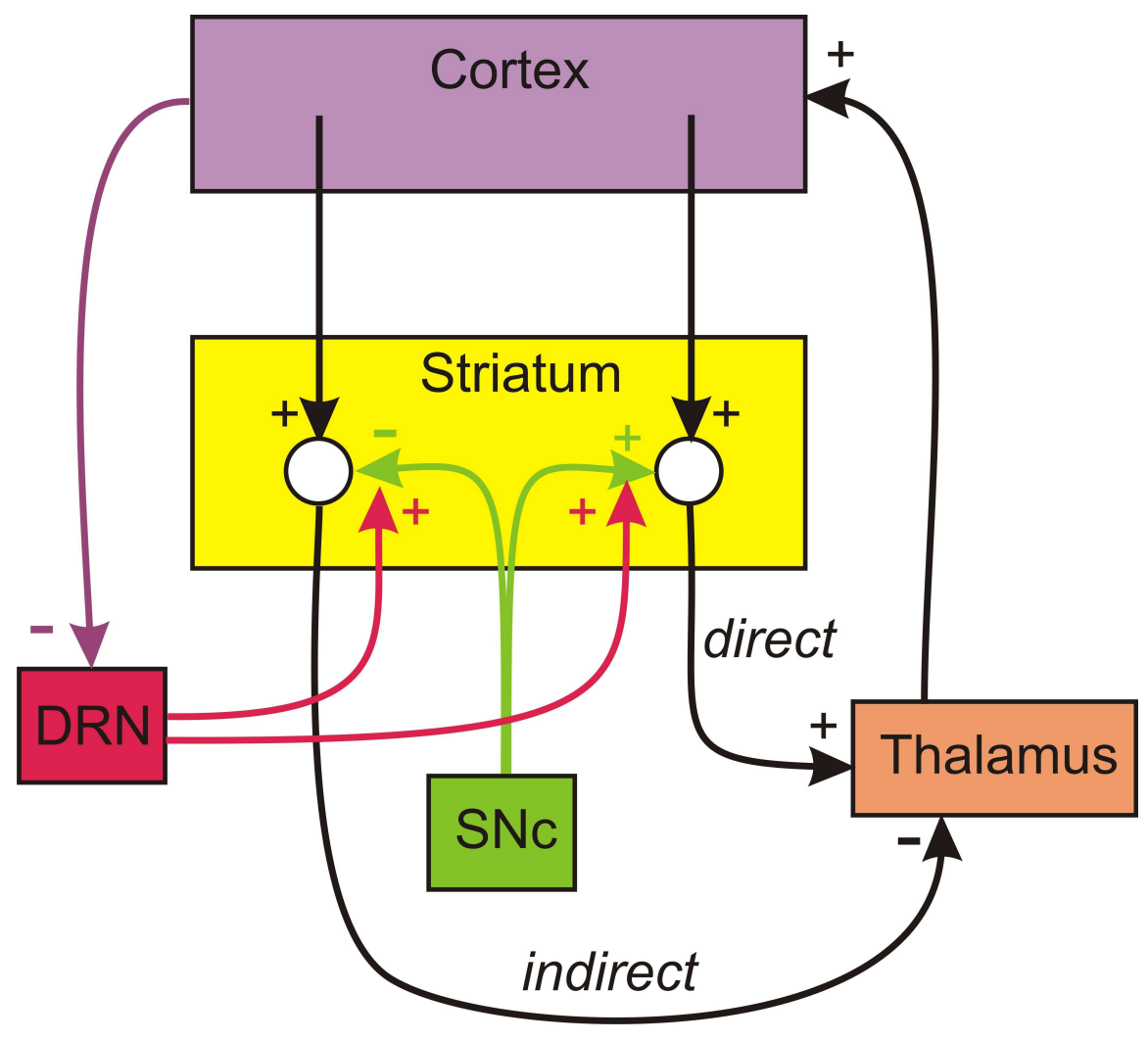

FiguRE 1. A schematic view of the basal ganglia. Abbreviations: DRN, dorsal raphe nucleus; SNc, substantia nigra pars compacta

Figure 1 and no inputs to the SNc. But, in fact, both nuclei receive many electrophysiological inputs from other brain regions. So the pharmacological network projects biochemical changes over long distances and the biochemical changes affect the electrophysiological network. And the electrophysiological network, in turn, projects to and affects the pharmacological network.

\section{Challenges for mathematical neuroscience.}

Most mathematical work in neuroscience has consisted of biophysical models for single neurons or network models that greatly simplify the individual neuron properties. The biophysical models have enabled us to understand the complicated firing patterns of individual neurons and how those patterns change as parameters are varied. The network models focus on how emergent group behavior, for example synchrony, arises from the pattern of connections, both excitatory and inhibitory, between the nodes in the network that represent individual neurons. Both kinds of models have helped to explain experimental data and have increased our understanding of the electrophysiological networks in the brain. The mathematical challenge is to expand these models to take account of the pharmacological network properties of the brain.

An excellent first step in integrating pharmacology into electrophysiological models would be to take a close look at the parameters whose bifurcations change the electrophysiological behavior of the system. Many of these parameters may, in fact, depend on pharmacological variables, some of them projected from distant locations. This is already done in biophysical models of single neurons where the ionic environment in the extracellular space affects the spiking behavior of the cell. The next natural step is 
to understand how the pharmacological environment affects the dynamics of local electrophysiological networks. This is really just a first step because the focus is still on the electrophysiological network; we are just including the possibility that its behavior is dependent on local pharmacology.

The example in the previous section (Figure 1) was overly simple in one important respect. The DRN and the SNc project DA and 5HT changes to the striatum and the DRN and the SNc are both influenced by their electrophysiological inputs. What was left out was that the DRN projects to the SNc and inhibits it [12] and the SNc projects to the DRN and excites it [8]. That is, there is a network of nuclei that project biochemistry over long distances. The nodes in this network are the nuclei themselves and we should draw edges when they influence each other. There is a pharmacological network that interacts with the electrophysiological network.

It's hard to see what a general mathematical theory would be that integrates electrophysiological and pharmacological networks. For the kinds of pharmacological networks described in the above example, the scales are too different. The nuclei that project biochemistry over long distances contain tens of thousands or hundreds of thousands of cells and they influence by volume transmission nuclei of similar size. The projection regions contain the local electrophysiological networks that are often the object of study in current mathematical neuroscience. Thus, the nodes of the pharmacological networks contain exceptionally large electrophysiological networks. In addition, the time scales are usually different. The time scales of action potentials are milliseconds or seconds, while the time scale of biochemical changes are typically seconds to minutes.

There are intermediate levels, however, where the scales might be similar. The basal ganglia is thought to be the site of action selection [11]. The idea is that parallel pathways through the basal ganglia code for different actions. The cortical input and processing in the basal ganglia determines which action is temporarily selected and represses others actions. Small local networks that accomplish this have been proposed and it is thought that the selection process involves a local increase in DA [11]. From this point of view, the diagram in Figure 1 refers only to the small sets of neurons in each nucleus that are involved in a particular action. In this case, the DA would diffuse over and influence only a very small portion of the striatum, so in this sense the scales of the volume transmission and the local electrophysiology would be similar. The review [10] emphasizes this local aspect of volume transmission. One would then view the entire cortical-basal ganglia-DRN circuit as consisting of a very large number of such interacting local pharmacological and electrophysiological networks in parallel.

It is important to remember how hard these questions really are. Of course, electrophysiology and pharmacology are important for understanding the brain. Equally important are genomics, behavior, and anatomy. By genomics, we don't mean the set of genes that each of us was given, but instead we mean the changing levels of gene expression in brain cells that are influenced by electrophysiology, pharmacology, and behavior through the endocrine system. And, by anatomy, we mean the morphology of individual cells, the connection patterns of neurons, and the proprioceptive feedback to the brain from the body [5]. In turn, behavior is influenced by electrophysiology, pharmacology, genomics and anatomy. All of these different aspects of the brain are dynamic, that is they are changing locally in time, and they all influence each other. Eventually mathematical neuroscience will have to invent structures that enable us to understand how these systems work together to make us who we are.

Acknowledgements. This research was partially supported by NSF grants EF-1038593 (HFN,MR), NSF agreement 0112050 through the Mathematical Biosciences Institute (JB, MR), an NSF CAREER Award (JB), the Alfred P. Sloan Foundation (JB), and NIH grant R01 ES019876 (D. Thomas).

\section{References}

[1] A. Adell, P. Celada, M. T. Abella, F. Artigasa. Origin and functional role of the extracellular serotonin in the midbrain raphe nuclei. Brain. Res. Rev. 39 (2002), 154-180.

[2] P. Blandina, J. Goldfarb, B. Craddock-Royal, J. P. Green. Release of endogenous dopamine by stimulation of 5-hydroxytryptamine3 receptors in rat striatum. J. Pharmacol. Exper. Therap., 251(1989), 803-809. 
[3] N. Bonhomme, P. Duerwaerdere, M. Moal, U. Spampinato. Evidence for 5-HT4 receptor subtype involvement in the enhancement of striatal dopamine release induced by serotonin: a microdialysis study in the halothane-anesthetized rat. Neuropharmacology, 34 (1995), 269-279.

[4] D. J. Brooks. Dopamine agonists: their role in the treatment of Parkinson's disease. J. Neurol. Neurosurg. Psychiatry, 68 (2000), 685-689.

[5] H. J. Chiel, R. D. Beer. The brain has a body: adaptive behavior emerges from interactions of nervous system, body, and environment. Trends Neuroscience, 20 (1997), 553-557.

[6] R. A. Cunha. Different cellular sources and different roles of adenosine: A1 receptor- mediated inhibition through astrocytic-driven volume transmission and synapse-restricted A2A receptor-mediated facilitation of plasticity. Neurochem. Int., 52 (2008), 65- 72 .

[7] P. Deurwaerdere, N. Bonhomme, G. Lucas, M. Moal, U. Spampinato. Serotonin enhances striatal overflow in vivo through dopamine uptake sites. J. Neurochem., 66 (1996), 210-215.

[8] V. DiMatteo, G. DiGiovanni, M. Pierucci, E. Esposito. Serotonin control of central dopaminergic function: focus on in vivo microdialysis studies. Prog. Brain Res., 172 (2008), 7-44.

[9] R. Feldman, J. Meyer, L. Quenzer. Principles of Neuropharmacology. Sunderland, MA.: Sinauer Associates, Inc., Sunderland MA, 1997.

[10] K. Fuxe, A. B. Dahlstrom, G. Jonsson, D. Marcellino, M. Guescini, M. Dam, P. Manger, L. Agnati. The discovery of central monoamine neurons gave volume transmission to the wired brain. Prog. Neurobiol., 90(2010), 82-100.

[11] C. R. Gerfen, D. J. Surmeier. Modulation of striatal projection systems by dopamine. Annu. Rev. Neurosci., 34(2011), 441-466.

[12] B. P. Guiard, M. E. Mansari, Z. Merali, P. Blier. Functional interactions between dopamine, serotonin and norepinephrine neurons: an in-vivo electrophysiological study in rats with monoaminergic lesions. Int. J. Neuropsycopharm., 11(2008), 625-639.

[13] J. P. Hornung. The human raphe nuclei and the serotonergic system. J. Chem. Neuroanat., 26(2003), 331-343.

[14] A. Lasota, M. Mackey. Probabilistic Properties of Deterministic Systems. Springer-Verlag, New York, 1985.

[15] J. Losson, M. Mackey. A Hopf-like equation and perturbation theory for delay differential equations. J. Stat. Phys., 69(1992), 1025-1046.

[16] M. C. Mackey. A unified hypothesis for the origin of aplastic anemia and haematopoiesis. Blood, 51(1978), 941-956.

[17] M. C. Mackey, L. Glass. Oscillations and chaos in physiological control systems. Science, 197(1977), $287-289$.

[18] M. C. Mackey, L. Glass. Pathological conditions resulting from instabilities in physiological control systems. Ann. N. Y . Acad. Sci., 316(1979), 214-235.

[19] M. C. Mackey, M. Tyran-Kaminska. Deterministic Brownian motion: The effects of perturbing a dynamical system by a chaotic semi-dynamical system. Physics Reports, 422(2006), 167-222.

[20] J. M. Monti. The structure of the dorsal raphe nucleus and its relevance to the regulation of sleep and wakefulness. Sleep Med. Rev., 14(2010), 307-317.

[21] M. Reed, H. F. Nijhout, J. Best. Computational Studies of the Role of Serotonin in the Basal Ganglia. Frontiers Integrative Neuroscience, 7(2013), 1-8.

[22] Y. Smith, M. Bevan, E. Shink, J. P. Bolam. Microcircuitry of the direct and indirect pathways of the basal ganglia. Neuroscience, 86(1998), 353-387. 